\title{
Dear Editor-in-Chief,
}

In Vol. 49 (4), 2011 of this journal, your review was published on the book „Statistics for Terrified Biologists“ written by van Emden. Your text excited in my mind two absolutely contradictory viewpoints.

First, I may only support the view of "terrified biologists" whenever they encounter mathematics. Hence, it seems reasonable to offer statistical treatments of biological experiments as simple as possible. But:

Second, such a procedure resembles users of mobile phones who are able to understand what key or button should be pressed in order to call certain person. In this communication, any understanding of the "mechanisms" of electronic transmission of words or pictures is of no importance. However, the use of statistical procedures could be crucial for the assessment of experimental results. It is generally acknowledged that results could very often enable quite dissimilar conclusions depending on the statistical method used and on the amount of energy invested into its processing and evaluation. By no means do I wish to say that experimental results in biology offer wilful conclusions depending only on the statistical processing! But even the simplest question: "Is my experimental treatment statistically significant or nonsignificant?" could often be answered differently when different statistics is used. For that matter also you emphasize the need to carry out experiments with sufficient number of replicates and to apply adequate statistical treatment.

My "second" continues. When I started my research carrier, the most tedious task was the calculation itself. Nowadays, computers need only to be fed with the data and subsequently statistical packages "do the work". But that is the point: Which procedure should be used and how to interpret the computed output? The best solution consists in the collaboration with a professional statistician. However, even then, the biologist should understand at least the principles of the applied statistics as well as limits of the statistical conclusions, meaning of individual statistical parameters etc. Similarly, it is invaluable if the statistician is reasonably familiar with the biological philosophy of the given experiment.

And my last "second" that I find the most important one. In biology, much research could be still done without mathematics. This is excellent news for all the students in my country, who are now allowed to pass their final examination at the secondary school and sidestep mathematics. Hence, basic and much of the applied research in biology will be more and more carried out by professional mathematicians or physicists and chemists. There are numerous examples of the necessity to apply mathematics in the understanding of biological systems. In the 1960s a canopy seemed to be too complicated for its quantitative description until mathematicians introduced acceptable assumptions and justified simplifications enabling the quantification of the canopy structure, microclimate, and several photosynthetic parameters. Let me recall just one name: Ju. Ross from Estonia. But the needs for the invasion of mathematics into biological research are numerous. Let us just imagine the understanding of photosynthesis at the cellular level. For example, an over-expression of genes for some enzymes becomes evident by assessing some of their products and/or processes. Experimentally, it is impossible to determine all the real or potential impacts on other processes in the same cell or even the tissue or the whole plant. The need of a comprehensive mathematical model is self-evident.

Dear Editor-in-Chief, I am aware of the fact that my stress on the education of mathematically literate biology students is by far not original. After all, much of our current knowledge on photosynthesis owes to the use of advanced mathematics in experimental research. I simply wish to express my view that scientists carrying experiments in biology do need and increasingly will need a deep understanding of mathematics. Biologists and students terrified by "mathematical formulae and mathematical thinking" will be more and more pushed to the edges of the creative research offering its unique beauty and magnificence of the human thinking.

L. NÁTR (Praha) 